\title{
Anatomy and function of the communication between knee joint and popliteal bursae
}

\author{
WOLFGANG RAUSCHNING \\ From the Department of Orthopaedic Surgery, University Hospital, S-75014 Uppsala, Sweden
}

SUMMARY The anatomy and function of the opening between the knee joint cavity and the gastrocnemio-semimembranosus bursa was studied in 120 necropsy specimens of knee joints both by conventional knife dissection and by a newly modified technique of serial cryosectioning of undecalcified joints frozen at various angles of flexion. The communication invariably took the shape of a transverse slit separating the capsule from the undersurface of the gastrocnemius tendon. On flexion it opened as the pull from the semimembranosus tendon and the medial meniscus widened the gap. On extension the communication was closed by compression by the overlying tendons. A functional closing action was invariably demonstrated, whereas no unidirectional valve mechanism was found.

In the 1840s Adams observed cystic swelling in the popliteal region of knees affected by 'chronic rheumatoid arthritis' so frequently that he considered it to be a reliable symptom of rheumatoid affection of the joint. He observed that the swelling was due to an enlargement of the bursa beneath the medial head of the gastrocnemius and that it communicated with the joint cavity by 'a species of valvular opening'. Poirier $^{3}$ also ascribed the popliteal cysts to fluid distension of the compound gastrocnemiosemimembranosus bursa, which he consequently called 'la bourse des kystes poplités'.

A different mode of popliteal cyst formation was proposed by Gruber, ${ }^{4}$ who considered that the enlarged bursae as well as the popliteal cysts resulted from protrusion of the synovial membrane through the fibrous capsule. His concept was adopted in Billroth's textbook, ${ }^{5}$ which was quoted by Baker. ${ }^{6}$ Although Baker was uncertain about the origin of the cysts whose clinical course he described in 2 classical papers, ${ }^{6,7}$ his name has since been eponymously used for popliteal cysts, especially those occurring in rheumatoid arthritis, commonly implying a hernial origin.

In 1856 Foucher $^{8}$ observed that by manual compression the fluid from the cyst could be forced into the joint when the knee was flexed, whereas this was never possible when the knee was extended. Although a true unidirectional valve mechanism is

Accepted for publication 22 August 1979 Correspondence to Dr Rauschning. operative in some patients, with constantly distended popliteal cysts impeding the flow of fluid, even when the knee joint is in flexion (negative sign of Foucher), communicating bursae can usually be emptied into the joint. This has frequently been demonstrated at arthrography, ${ }^{9}$ with simultaneous recording of fluid pressures developing in the joint cavity and the bursa in various knee positions and during the use of the knee joint. ${ }^{10,11}$

Considering the high frequency of the communication and its importance in the formation of popliteal cysts, very little has been reported on its morphology and function in the main textbooks of anatomy.

\section{Materials and methods}

At necropsy 120 fresh knee joint specimens were selected from subjects for whom a study of the medical records and inspection revealed no history of joint disease or knee surgery. Sixteen knees were from subjects younger than 50 years of age and the remainder from older subjects.

\section{KNIFE DISSECTION}

Conventional knife dissection was carried out on 108 knees. With the body lying prone the posteromedial aspect of the knee capsule was exposed by resection of the gastrocnemius, semimembranosus, and semitendinosus tendons. The frequency of a communicating opening, its shape and size, and its topographic location were studied, as well as the 
deformations of the orifice occurring on simulated pull of the tendons and on extension and rotation of the joint. The whole capsule overlying the medial femoral condyle was cut out, inspected from within, and searched for weak portions by fibre-light transillumination.

\section{SERIAL CRYOSECTIONING OF}

\section{UNDECALCIFIED S PECIMENS}

Twelve knees with communicating popliteal bursae were filled with $60-80 \mathrm{ml}$ of water stained with methylene blue and frozen in situ at various angles of flexion or in extension by repeated application of carbon dioxide snow. The specimen was then cut out and embedded and frozen on a large microtome stage containing carboxymethyl-cellulose. Specimens were cut in the horizontal or sagittal planes on a heavyduty cryomicrotome (LKB 2250 PMW, Bromma, Sweden). Sections 20-50 $\mu \mathrm{m}$ thick were cut from the block, and at intervals of $1 \mathrm{~mm}$ the surface of the specimen contained in the block was thawed and

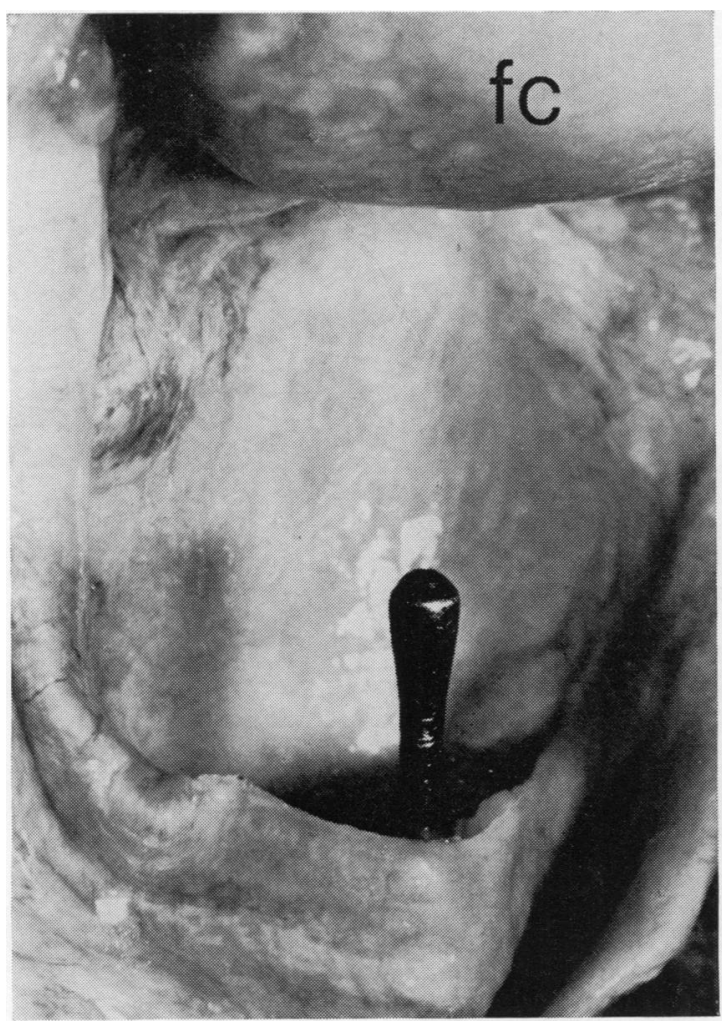

Fig. 1 Intra-articular view of the posteromedial joint capsule. Femoral condyle $(f c)$ above. A probe is inserted into the broad capsular gap. photographed. This modification of the technique of large-specimen cryosectioning ${ }^{12}$ prevented deformations which might have resulted from conventional collection of the sections on tape or glass plates and the subsequent histological procedures of fixation and staining.

\section{Results}

\section{KNIFE DISSECTIONS}

In 58 of the 108 knees the gastrocnemio-semimembranosus bursa communicated with the knee joint cavity. The capsular opening invariably had the shape of a transverse slit opposite the upper lateral circumference of the medial femoral condyle and $2 \cdot 0-5 \cdot 5 \mathrm{~cm}$ distal to the origin of the gastrocnemius tendon (Fig. 1). The lower rim of the slit was sharp, whereas no definite upper rim was found on the broad arch of the gastrocnemius tendon lying behind and above the slit, which in the mediolateral direction measured 4-24 mm (mean $18 \mathrm{~mm}$ ).

In 50 specimens no communication was found. At transillumination a constant semicircular area of capsular thinning was seen distal to the straight line at which the gastrocnemius tendon merged with the capsule. This weak portion measured $1-2 \mathrm{~cm}^{2}$. In some cases the junction between the capsule and the gastrocnemius tendon was extremely thin and

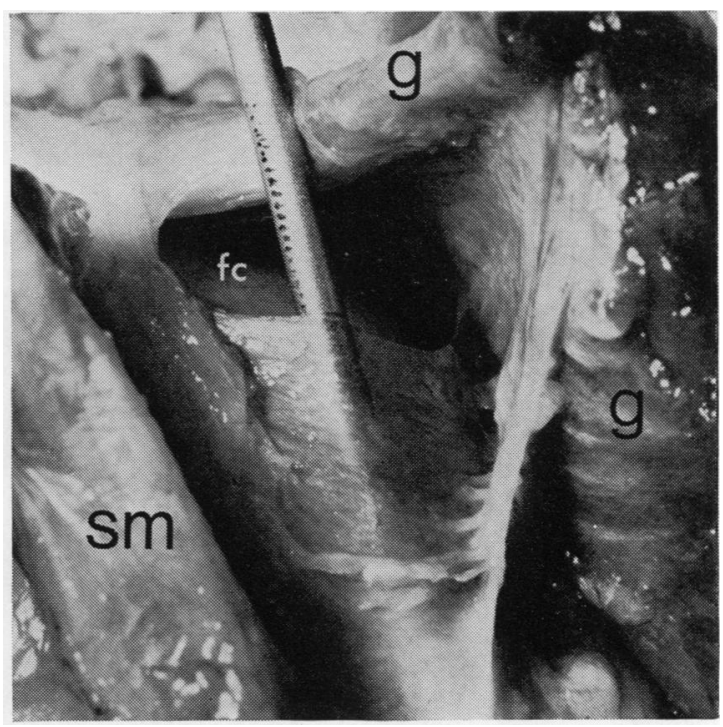

Fig. 2 Extra-articular view of the communication site.

The tendons of the gastrocnemius $(g)$ and semimembranosus (sm) muscles are divided and retracted. Note the thin upper rim of the capsule and the underlying femoral condyle $(f c)$. 
ruptured during the dissection. No other weak areas were observed in the capsule. When a slit-shaped communication was found, the capsule was always very thin and transparent adjacent to its upper rim (Fig. 2), which had the appearance of a heart valve.

Rotation of the tibia caused diagonal deformations of the slit, especially on slight flexion of the knee, and pulling on the semimembranosus and gastrocnemius tendons markedly widened the gap in the craniocaudal direction, especially with the greater degrees of knee flexion. The semimembranosus, by the action of its capsular arm (i.e., the oblique popliteal ligament), pulled the capsule downwards.

CRYOSECTIONS

With increasing degrees of knee flexion the communication became progressively wider as the
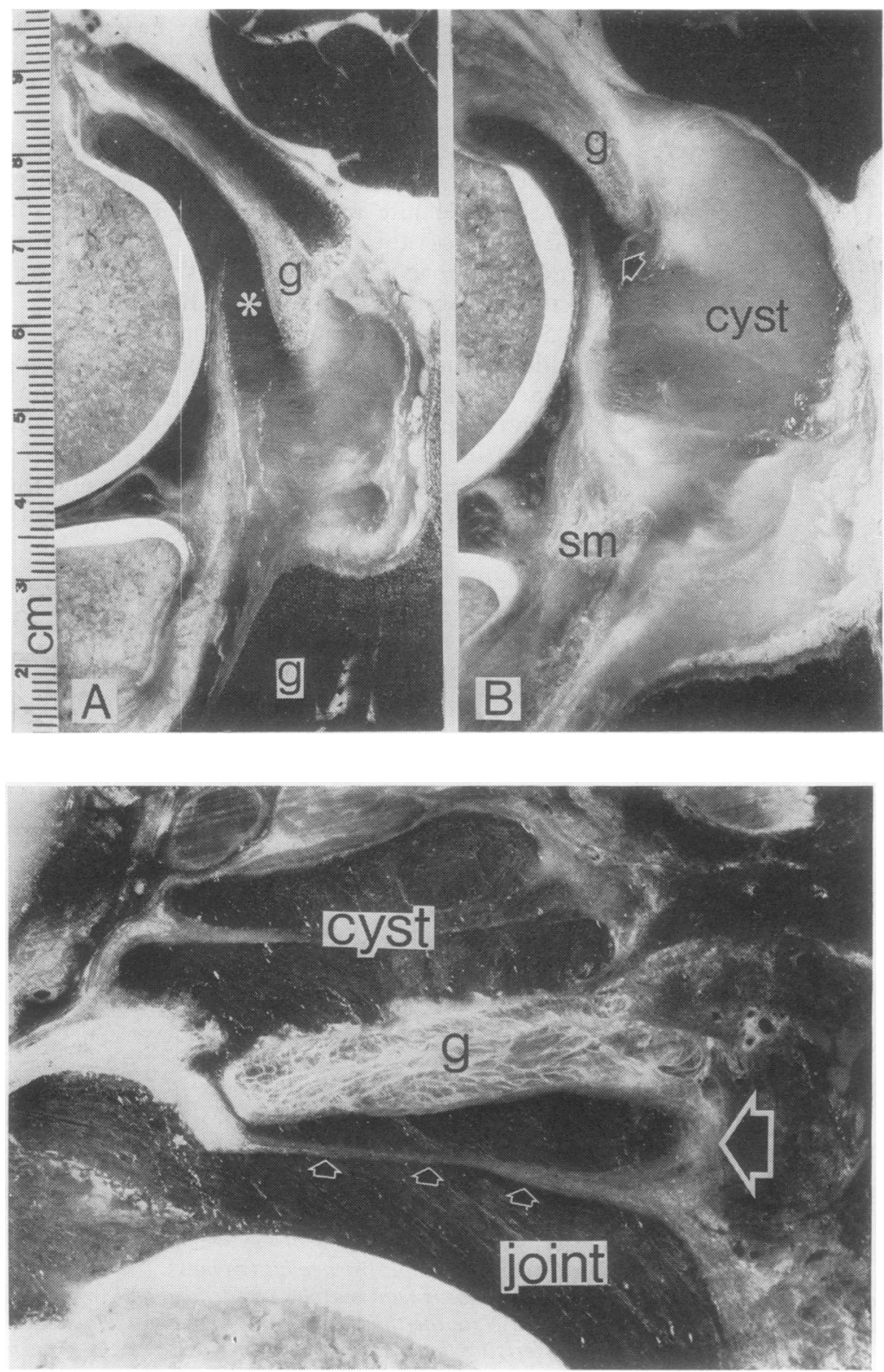

Fig. 3 Two sagittal cryosections. A: through the lateral portion of the capsular opening; B: at its medial edge. The large cyst displaces the tendons. The asterisk indicates the canal into the joint cavity.

Fig. 4 Horizontal cryosection at the level of the thin upper capsular rim (small arrows). Note the thickness of the gastrocnemuis tendon $(\mathrm{g})$. The large arrow indicates why on lateral arthrograms the canal may be misinterpreted as a narrow neck. 


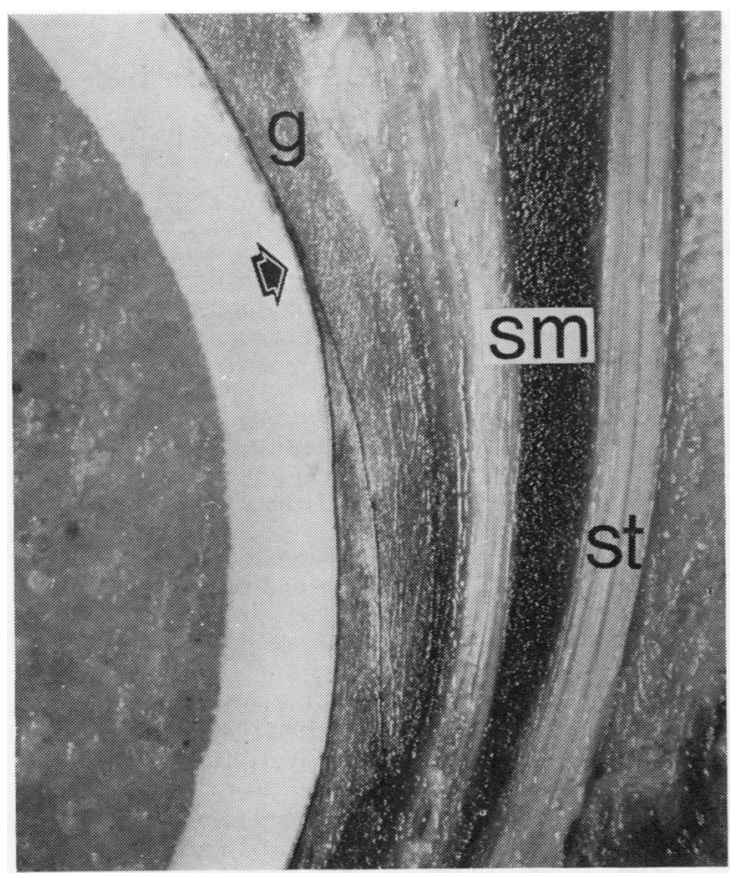

Fig. 5 Detail from a sagittal plane cryosection of a knee joint specimen which was frozen at extension. The thin upper rim of the capsule (arrow) is firmly compressed between the femoral condyle and the overlying tendons of the gastrocnemius $(g)$, semimembranosu: (sm), and the semitendinosus (st) muscles.

gastrocnemius tendon retracted dorsally away from the subjacent femoral condyle (Fig. 3). In horizontal sections the portion of the bursa situated between the gastrocnemius tendon and the capsule was filled with fluid (Fig. 4).

On extension of the knee the broad tendinous arch of the gastrocnemius muscle was first firmly stretched over the upper convexity of the femoral condyle, and on further extension the upper rim of the capsule was compressed between the tendon and the condyle. When the knee was extended yet further, the fluid from the posterior recess of the knee as well as from the subgastrocnemial portion of the bursa was progressively emptied, and on final extension the heavy tendons of the semimembranosus and semitendinosus muscles, owing to their divergent directions of pull, were firmly twisted around the medial and posterior aspects of the gastrocnemius tendon. This compression between the tendons appeared to afford a further degree of closure and obviously increased the compression of the capsular rim (Fig. 5). In horizonal sections the posterior portion of the bursa bulged maximally beneath the popliteal fascia on full extension.

\section{Discussion}

Communication between the joint cavity and the gastrocnemio-semimembranosus bursa was found in over $50 \%$ of cases, a figure consistent with the findings of previous investigators. ${ }^{13}$ None of the other posteromedial bursae communicated with the joint. The opening displayed constant anatomical features with respect to location, shape, and width. Lindgren and Willén ${ }^{14}$ found that the communication was rare in younger subjects owing to the interposition of a broad layer of elastic connective tissue between the joint and the bursa. During aging progressive rarefaction and thinning of this barrier occurred. The results of this study favour the view that the degenerative changes at the junction between the capsule and the gastrocnemius tendon are due to the capsular tearing forces emanating from the pull of the meniscus and the semimembranosus tendon. The progressive thinning and the final communication would consequently represent a physiological, acquired condition.

The alternating opening and closing of the communication provides a morphological explanation for Foucher's sign and also explains why the vast majority of communicating popliteal bursae which are accidentally detected at arthrography ${ }^{9}$ do not develop into clinically significant and palpable popliteal cysts.

By means of simultaneous recording of the intraarticular and intrabursal fluid pressures occurring in knees with chronic or simulated effusions during joint use, Jayson and Dixon ${ }^{10}$ convincingly demonstrated that a considerable pressure rise occurs in the joint as well as in the bursa during knee bending and squatting and that no pressure rise is transmitted between these cavities when the knee is extended. The present study provides a morphological explanation for these pressure changes. During knee bending with weight bearing, for example during the stance phase of gait, the fluid pressures are extremely high in the joint as well as in the communicating bursa $^{15}$ During extension the communication is suddenly blocked, entrapping the fluid at this high pressure. Further extension of the knee appears to cause even higher intrabursal pressures as the deep portions of the bursa are firmly compressed by the cross-action of the overlying tendons (Fig. 5).

This mechanism would make it understandable why patients have most discomfort from their popliteal cysts during extension of the knee and why they usually experience relief when the knee is flexed, as, for example during the swing phase of gait. 
In this phase the opening of the communication allows the fluid pressures to equilibrate by reflux of fluid to the joint. This closing mechanism is consistent with the significantly higher fluid pressures in the cyst and the fact that the volume of joint effusion is significantly smaller in knee joints with a communicating cyst. ${ }^{10}$ As the entrapment of fluid in the cyst is not due to an anatomical structure causing a true, unidirectional obstruction of flow in either direction even during knee flexion, it should be referred to as a 'functional valve mechanism'.

The valve-like aspect of the communication (Figs. 1 and 2) has caused some authors to assume that a true valvular mechanism is constantly operative at this location. ${ }^{2,16}$ But if the free rim of the capsule was forced against the gastrocnemius it would rather impede flow from the joint to the bursa.

Another common morphological misinterpretation is the existence of a curved narrow neck, pedicle, or stalk between the joint cavity and the bursa. This idea has arisen from the shape of the communication on lateral arthrograms (it is not visible in the frontal view, as it is concealed by the femoral condyle and the contrast medium in the joint and the bursa). As seen in Figs. 3A and 4 the canal (i.e., the subgastrocnemial portion of the bursa) is a cavity, $2-3 \mathrm{~cm}$ broad the walls of which are fairly rigid and straight and cannot be expected to collapse to form a valve of the Bunsen type. The alternating closing and opening of the communication would explain why, during pressure measurements, Lindgren ${ }^{11}$ did not find a progressive increase in pressure in the bursae as would be expected if a true valve mechanism was present. The observation of Pinder ${ }^{17}$ in the course of 14 anterior synovectomies that all popliteal cysts could be emptied into the joint cavity even though they contained fibrin masses is in accordance with own observations, and it indicates a fairly wide communication. Nor does it suggest a true valvular mechanism of the ball type in all rheumatoid cases.
References

1 Adams R. Abnormal conditions of the knee joint. $\stackrel{\oplus}{\rightarrow}$ Anat Physiol(Lond) 1839, 1847; 3: 48-78.

2 Adams R. Chronic rheumatic arthritis of the knee joint. $\frac{\overline{ }}{\bar{N}}$ Dublin J Medi Sci 1840; 17: 520-522.

3 Poirier P. Bourses sèreuses du genou (règion postérieure). Bourses séreuses de la région poplitèe. Arch Gén Méd
$1886 ; 38: 539-575$.

4 Gruber W. Ueber die Ausstuelpungen der Synovialkapsel $\vec{\circ}$ des Kniegelenkes und ueber die chirurgische Wichtigkeit der Communication derselben mit einigen benachbarten $\overrightarrow{\vec{\omega}}$ Schleimbeuteln. Vierteljahres prakt Heilk 1845; 5: 95-105.

5 Billroth T. General Surgical Pathology and Therapeutics,

trans. C E Hackley. London: Lewis, 1875 . in connection with disease of the knee joint. St Bart Hosp Rep 1877; 13: 245-261.

7 Baker W M. The formation of abnormal synovial cysts in connection with the joints (second communication). St 음 Bart Hosp Rep 1885; 21 : 177-190.

8 Foucher E. Mèmoire sur les kystes de la règion poplitèe. Arch Gén Méd 1856; 8: 313-335.

- Doppman J L. Baker's cyst and the normal gastrocnemiosemimembranosus bursa. AJR 1965; 94: 646-652.

10 Jayson M I V, Dixon A ST J. Valvular mechanisms in $\overrightarrow{0}$ juxta-articular cysts. Ann Rheum Dis 1970; 29: 415-420.

11 Lindgren P -G. Gastrocnemio-semimembranosus bursa. and its relation to the knee joint. III Pressure measurements in joint and bursa. Acta Radiol (Diagn) (Stockh) 1978; 19: 377-388.

12 Ullberg S. The technique of whole body autoradiography. Cryosectioning of large specimens. Sci Tools (special ڤ̆ issue) 1977; 2: 2-29.

13 Wilson P D, Eyre-Brook A L, Francis J D. A clinical and anatomical study of the semimembranosus bursa in 0 relation to popliteal cyst. $J$ Bone Joint Surg 1938; 20: 963-984.

14 Lindgren P -G, Willén R. Gastrocnemio-semimembranosus bursa and its relation to the knee joint. I. Anatomy and histology. Acta Radiol (Diagn) (Stockh) 1977; 18 : 497-512.

15 Dixon, A St J, Grant C. Acute synovial rupture in rheumatoid arthritis. Clinical and experimental observations. Lancet $1964 ; 1$ : 742-745.

16 Taylor A R, Rana N A. A valve. An explanation of the formation of popliteal cysts. Ann Rheum Dis 1973; 32:0 419-421.

17 Pinder I M. Treatment of the popliteal cyst in the rheumatoid knee. J Bone Joint Surg 1973; 55B: 119-125. ․ㅡ. 